\title{
Determinación de Habilidades Cognitivas en Distintas Asignaturas Clínicas del Departamento de Odontología, Universidad de Antofagasta, Chile
}

\author{
Determination of Cognitive Skills in Differents Clinics \\ Courses of Dentistry of the University of Antofagasta, Chile
}

Bravo-Bown, J.; Chung, C.; García, F.;; Nikolic, L.* \& Pizarro, C.*

BRAVO-BOWN, J.; CHUNG, C.; GARCÍA, F.; NIKOLIC, L. \& PIZARRO, C. Determinación de habilidades cognitivas en distintas asignaturas clínicas del Departamento de Odontología, Universidad de Antofagasta, Chile. Int. J. Odontostomat., 10(2):309-313, 2016.

RESUMEN: El objetivo fue determinar las habilidades cognitivas más frecuentemente evaluadas en las pruebas escritas de final de unidad aplicadas durante el año 2013, en algunas asignaturas clínicas del Departamento de Odontología de la Universidad de Antofagasta. Se realizó un estudio de naturaleza cuantitativa, de diseño observacional, prospectivo, transversal y analítico que analizó las pruebas escritas de final de unidad en las asignaturas de periodoncia, rehabilitación, operatoria, cirugía y endodoncia en el Departamento de Odontología de la Universidad de Antofagasta. En el análisis y determinación de las habilidades cognitivas de cada pregunta participaron dos evaluadores en forma independiente. Se utilizó un protocolo de determinación de habilidades cognitivas, instrumento que fue validado por expertos. Las observaciones realizadas fueron promediadas y expresadas según frecuencias relativas (\%), se utilizó software SPSS (v.22). El 95,8 \% de las preguntas analizadas evaluaron habilidades cognitivas inferiores y el $4,12 \%$ superiores. La mayor proporción de las preguntas analizadas corresponden al nivel cognitivo conocimiento $(71,9 \%)$ y la menor al de la evaluación (1,5\%). De las 5 asignaturas analizadas las que desarrollan más frecuentemente habilidades cognitivas superiores son periodoncia (12\%), endodoncia $(3,3 \%)$ y operatoria $(2.1 \%)$.

PALABRAS CLAVE: educación superior, educación en odontología, evaluación.

\section{INTRODUCCIÓN}

El mundo actual está pasando de una sociedad basada en la industria productiva a otra basada en el conocimiento, pasando por un estado intermedio que es el de la sociedad de la información (Álvarez de Eulate \& Villardón Gallego, 2006). En los tiempos actuales y futuros, el desarrollo de habilidades que les permitan a los profesionales acceder a la información a través de la tecnología, lograr su comprensión y comunicarla en forma efectiva (Habilidades TIC) es una necesidad. Para las universidades constituye un reto y un motivo de cambio el ajustarse a las demandas de la sociedad del siglo XXI (Bricall \& Brunner, 2000). En las instituciones educativas universitarias es común encontrar asignaturas que replican los modelos de enseñanza de antaño, desarrollando clases magistrales en las cuales el profesor entrega el conocimiento de manera presencial y los estudiantes se preocupan de tomar apuntes lo más concentradamente posible, normalmente después de 4 o 5 clases magistrales, se realiza un control escrito que pretende evaluar el aprendizaje de los contenidos entregados. No obstante en los tiempos actuales la evaluación debe estar centrada no en "la medición" sino en "la retroalimentación" la que permite facilitar la toma de decisiones y mejorar el proceso de enseñanza. El aprendizaje debe estar centrado no en la memorización de los contenidos sino en la aplicación de los mismos, en su análisis, su discusión, etc. Sumado a lo anterior la gran mayoría de los académicos que trabajan en los Departamentos o Facultades de Odontología a nivel nacional e internacional no son "profesores de profesión" sino "cirujanos-dentistas", y realizan habitualmente sus labores 
como docente replicando la forma o los métodos a través de los cuáles ellos mismos fueron formados, esto que puede vislumbrarse como una fortaleza, también puede ser considerado una debilidad si es que no se actualizan los conocimientos y las formas de enseñanza.

Las habilidades cognitivas son operaciones del pensamiento por medio de las cuales el sujeto puede apropiarse de los contenidos y del proceso que usó para ello. Son un conjunto de operaciones mentales cuyo objetivo es que el alumno integre la información adquirida, básicamente a través de los sentidos, en una estructura de conocimiento que tenga sentido para él (Laorden et al., 2005). El año 1956 el Dr. Benjamín Bloom creó un sistema de clasificación o taxonomía que ordenó y jerarquizó los objetivos del proceso de aprendizaje. Su clasificación es un continuo que parte de habilidades de pensamiento de orden inferior (conocimiento, comprensión y aplicación) y va hacia habilidades de pensamiento de orden superior (análisis, síntesis, evaluación). Se basa en la idea de que las habilidades cognitivas pueden clasificarse en seis niveles de complejidad creciente. Cada nivel depende de la capacidad del alumno para desempeñarse en el nivel o los niveles precedentes. Por ejemplo, la capacidad de evaluar, el nivel más alto de la taxonomía cognitiva, se basa en el supuesto de que el estudiante, tiene que disponer de la información necesaria (conocimiento), comprender esa información, ser capaz de aplicarla, de analizarla, de sintetizarla y, finalmente de evaluarla (Bloom, 1971).

La evaluación es clave en cualquier proceso educativo, y debe estar planificada de manera de favorecer el proceso de enseñanza y desarrollar no solo las habilidades cognitivas de orden inferior sino en forma preponderante las de orden superior. Los docentes deben por lo tanto actualizar sus métodos de enseñanza de manera de poder entregar a los estudiantes las herramientas que les permitan el análisis, la síntesis y la evaluación de los conocimientos. Por todo lo antes mencionado es sumamente relevante analizar los procesos evaluativos en las Instituciones de educación superior, específicamente en lo referente a la construcción y aplicación de los instrumentos evaluativos.

En esta investigación se determinaron las habilidades cognitivas más frecuentemente evaluadas en las pruebas escritas de final de unidad aplicadas durante el año 2013 en el Departamento de Odontología de la Universidad de Antofagasta en las asignaturas clínicas de periodoncia, endodoncia, rehabilitación, cirugía y operatoria.

\section{MATERIAL Y MÉTODO}

Se realizó un estudio de naturaleza cuantitativa, de diseño observacional, prospectivo, transversal y analítico que analizó las pruebas escritas de final de unidad aplicadas durante el año 2013 en las asignaturas de periodoncia, rehabilitación, operatoria, cirugía y endodoncia en el Departamento de Odontología de la Universidad de Antofagasta. En el análisis y determinación de las habilidades cognitivas de cada pregunta participaron dos evaluadores en forma independiente. Se utilizó un protocolo de determinación de habilidades cognitivas, instrumento que fue validado por expertos.

Validación de Instrumento. Los expertos que participaron en la validación del instrumento de medición fueron: Velia del Carmen Romero Torrejón, Magister en Gestión y Planificación Educacional; Carlos Alberto Muñoz Sánchez, Magister en Curriculum y Evaluación; Priscila Puentes Reyes, Magister en Educación, Doctorado en Educación; Kattherine Maricel Urriola López. Profesora de Español, Licenciada en Educación, Máster en Investigación Didática, Formación y Evaluación Educativa. Doctora en Educación por la Universidad de Barcelona; Mario Tapia Henriquez. Profesor en Español, Licenciado en Educación, Máster en Investigación Didáctica, Formación y Evaluación Educativa, Doctor en Educación por la Universidad de Barcelona. Cada experto realizó el análisis del instrumento de manera individual y determinó con respecto a cada indicador la univocidad (si/no), la importancia y la pertinencia (muy baja, baja, moderada, alta, muy alta). Se registró cada una de las observaciones y análisis de los expertos de manera de optimizar el instrumento de medición.

Plan de Análisis de los Datos. Las observaciones realizadas por los evaluadores fueron promediadas y expresadas según frecuencias relativas (\%) (Tabla I). Se utilizó software SPSS (v.22) y planillas excel.

\section{RESULTADOS}

Según nivel cognitivo. El $95,8 \%$ de las preguntas analizadas evaluaron habilidades cognitivas inferiores y el $4,12 \%$ a las superiores (Tabla I).

Según habilidad cognitiva. La mayor proporción de las preguntas analizadas corresponden a la determi- 
nación del nivel cognitivo conocimiento $(71,9 \%)$ y la menor al nivel cognitivo de la evaluación (1,5\%) (Tabla II).

Según asignatura. De las 5 asignaturas analizadas las que desarrollan más frecuentemente habilidades cognitivas superiores son periodoncia (12\%), endodoncia $(3,3 \%)$ y operatoria $(2,1 \%)$ (Tabla III). Las asignaturas que más frecuentemente evalúan la habilidad cognitiva de evaluación es periodoncia $(3,1$ $\%)$, rehabilitación $(1,9 \%)$ y endodoncia $(1,1 \%)$ (Tabla IV).

Tabla I. Distribución según nivel de habilidades cognitivas.

\begin{tabular}{lcc}
\hline \multirow{2}{*}{ Habilidades Cognitivas } & \multicolumn{2}{c}{ Preguntas analizadas } \\
\cline { 2 - 3 } \multicolumn{1}{c}{ Inferiores } & $\mathbf{n}$ & $\%$ \\
Superiores & 581 & 95,8 \\
Total & 25 & 4,1 \\
& 606 & 100 \\
\hline
\end{tabular}

Tabla II. Distribución según tipo de habilidad cognitiva.

\begin{tabular}{lcc}
\hline \multirow{2}{*}{ Habilidades Cognitivas } & \multicolumn{2}{c}{ Preguntas analizadas } \\
\cline { 2 - 3 } & $\mathbf{n}$ & $\mathbf{\%}$ \\
\hline Conocimiento & 435,5 & 71,9 \\
Comprensión & 135,5 & 22,4 \\
Aplicación & 10,0 & 1,7 \\
Análisis & 11,5 & 1,9 \\
Síntesis & 4,5 & 0,7 \\
Evaluación & 9,0 & 1,5 \\
Total & 606 & 100 \\
\hline
\end{tabular}

Tabla III. Distribución de los niveles de habilidades cognitivas según asignatura.

\begin{tabular}{lccccc}
\hline $\begin{array}{l}\text { Habilidades } \\
\text { Cognitivas }\end{array}$ & Periodoncia & Endodoncia & Rehabilitación & Operatoria & Cirugía \\
\hline Inferiores & 88,0 & 96,7 & 98,1 & 97,9 & 99,4 \\
Superiores & 12,0 & 3,3 & 1,9 & 2,1 & 0,6 \\
\hline
\end{tabular}

Tabla IV. Distribución de los tipos de habilidades cognitivas según asignatura.

\begin{tabular}{lccccr}
\hline & Periodoncia & Endodoncia & Rehabilitación & Operatoria & Cirugía \\
\hline Conocimiento & 53,9 & 60,6 & 79,8 & 79,2 & 83,9 \\
Comprensión & 31,8 & 36,1 & 14,8 & 18,8 & 14,9 \\
Aplicación & 2,3 & 0,0 & 3,6 & 0,0 & 0,6 \\
Análisis & 6,6 & 2,2 & 0,0 & 0,8 & 0,0 \\
Síntesis & 2,3 & 0,0 & 0,0 & 0,8 & 0,6 \\
Evaluación & 3,1 & 1,1 & 1,9 & 0,4 & 0,0 \\
\hline
\end{tabular}

\section{DISCUSIÓN}

La evaluación es la piedra angular en todo proceso educativo. Los procesos de medición y de evaluación en el ejercicio académico consumen una buena porción del tiempo del profesor. De acuerdo a Shaefer (1997) y Lissitiz (1991) (citados en Arends, 2007) los profesores pasan cerca del $10 \%$ de su tiempo en cuestiones relacionadas con la medición y la evaluación. Stiggins (2004), mencionó que los profesores pueden pasar tanto como un tercio de su tiempo en actividades "relacionadas con la evaluación". Es indispensable que los profesores desarrollen un repertorio de estrategias efectivas para evaluar y valorar a sus estudiantes. La manera en que se llevan a cabo las evaluaciones en el salón de clases tiene un efecto directo sobre la manera en que los estudiantes estudian y lo que aprenden (Crooks, 1988; Airasian, 2005; Brookhart, 2003). Existe bastante acuerdo que el aprendizaje tiene lugar cuando la persona sabe lo que se debe lograr, busca las estrategias para obtener este logro, y puede estimar el progreso que hace hacia la consecución del objetivo propiamente tal (Angelo \& Cross, 1993; Palomba \& Banta, 1999). Los estudiantes pueden dirigir y reorientar su propio aprendizaje, sólo cuando saben lo que constituye un buen desempeño (conocen los criterios de excelencia), son capaces de estimar su propio nivel de desempeño y 
pueden comparar éste con los criterios. La práctica evaluativa se centra exclusivamente en la determinación del nivel de logro, lo que constituye una condición necesaria, pero no suficiente. El alumno no solo requiere conocer la información de lo que ha logrado, sino que además necesita saber cómo y porqué se logra un determinado nivel (Himmel, 2011). Bajo esta perspectiva es deseable que los académicos puedan planificar y desarrollar estrategias que permitan fortalecer el conocimiento en sus estudiantes, en especial que logren en ellos el desarrollo de habilidades cognitivas de orden superior, que les permita el análisis, la síntesis y la evaluación de los conocimientos, solo a través de estos medios es posible que los estudiantes logren hacer frente a los desafíos actuales y futuros que la sociedad del conocimiento y de la información les demandarán. Ríos (2000) refiere que existe cada vez mayor consenso según el cual el sistema educativo no está cumpliendo efectivamente el cometido de formar a las futuras generaciones en las capacidades que necesita el ciudadano para desenvolverse en una sociedad que se transforma profunda y rápidamente. Se critica la falta de pertinencia de la formación que reciben los estudiantes con respecto a los retos que deben enfrentar en la sociedad, por lo cual la educación formal tiene la necesidad de reorientar sus objetivos y sus prácticas, a fin de responder a las nuevas demandas y proporcionar a los estudiantes una preparación más sólida y flexible que les permita una mejor adaptación a sistemas productivos sujetos a cambios rápidos, característicos de las sociedades actuales, donde los aprendizajes son contínuos, cambiantes, diversos y contextuales.

Es necesario que cada Institución educativa logre capacitar, normar y protocolizar todo lo referente a la evaluación de manera de favorecer el proceso de enseñanza y lograr un mayor aprendizaje. La evaluación en el aula es un componente consustancial al proceso de enseñanza y aprendizaje, ya que proporciona información a los estudiantes acerca de sus progresos en el aprendizaje y a la vez (dado que la evaluación es diseñada y analizada por los docentes), entrega a estos últimos los elementos de análisis necesarios para juzgar la eficacia de la docencia impartida (Himmel).

Es un hecho común que los profesores de educación superior supongan que sus estudiantes aprenden lo que ellos tratan de enseñarles spero a menudo encuentran evidencias decepcionantes, al examinar los resultados de las evaluaciones, comprobando que éstos no han aprendido de acuerdo a sus expectati- vas. Cuando los docentes advierten la brecha entre sus expectativas de aprendizaje y lo realmente aprendido por los estudiantes, puede ser demasiado tarde para remediar los vacíos detectados. A fin de evitar este desconcierto, tanto los docentes como los dicentes requieren formas más apropiadas y dinámicas para monitorear el aprendizaje durante los periodos académicos (Himmel). Es imprescindible seguir estudiando el tema de la evaluación en la educación superior con el fin de implementar estrategias de mejora que permitan fortalecer el proceso de enseñanza.

\section{CONCLUSIONES}

Las habilidades cognitivas preferentemente evaluadas son las inferiores (95,8 \%). El conocimiento o memorización fue la habilidad más frecuentemente evaluada $(71,9 \%)$, seguida por la comprensión $(22,4$ $\%)$. La aplicación, análisis y evaluación son evaluadas en menos del $2 \%$ de las veces y la síntesis es la habilidad cognitiva menos evaluada $(0,7 \%)$. La asignatura de periodoncia es la que más frecuentemente evalúa habilidades cognitivas de orden superior (12 $\%)$, seguida por endodoncia (3,3\%). La aplicación es más frecuentemente evaluada en la asignatura de rehabilitación (3,6\%). La comprensión es más frecuentemente evaluada en la asignatura de endodoncia $(36,1 \%)$.

BRAVO-BOWN, J.; CHUNG, C.; GARCÍA, F.; NIKOLIC, L. \& PIZARRO, C. Determination of cognitive skills in differents clinics courses of dentistry of the University of Antofagasta, Chile. Int. J. Odontostomat., 10(2):309-313, 2016.

ABSTRACT: The aims was to determine cognitive skills most frequently evaluated in the written tests final drive applied during 2013, in some clinicalcourses in the Department of Dentistry at the University of Antofagasta. A quantitative study of an observational, prospective, transversal and analytical which analyzed the written tests final drive in the courses of Periodontics, Rehabilitation, Operative, Surgery and Endodontics in the Department of Dentistry the University of Antofagasta. In the analysis and determination of the cognitive abilities of each question two evaluators participated independently. A protocol for determining cognitive abilities, an instrument that was validated by experts was used. The observations made by the evaluators were averaged and expressed as relative frequencies (\%), was used SPSS software (v.22). $95.8 \%$ of the questions analyzed evaluated lower cognitive abilities and $4.12 \%$ higher. The largest proportion of the questions analyzed correspond to the cognitive and knowledge level $(71.9 \%)$ lower than the 
BRAVO-BOWN, J.; CHUNG, C.; GARCÍA, F.; NIKOLIC, L. \& PIZARRO, C. Determinación de habilidades cognitivas en distintas asignaturas clínicas del Departamento de Odontología, Universidad de Antofagasta, Chile. Int. J. Odontostomat., 10(2):309-313, 2016.

evaluation (1.5\%). Of the 5 subjects analyzed which develop most often they are Periodontology higher Cognitive Skills (12\%), Endodontics (3.3\%) and Operatoria (2.1\%).

KEY WORDS: higher education, education in dentistry, evaluation.

\section{REFERENCIAS BIBLIOGRÁFICAS}

Airasian, P. W. Classroom Assessment: Concepts and Applications. New York, McGraw-Hill, 2005.

Álvarez de Eulate, C. Y. \& Villardón Gallego, L. Planificar desde Competencias para Promover el Aprendizaje: El Reto de la Sociedad del Conocimiento para el Profesorado Universitario. Bilbao, Universidad de Deusto, 2006.

Angelo, T. A. \& Cross, K. P. Classroom Assessment Techniques: A Handbook for College Teachers. $2^{\mathrm{a}}$ ed. San Francisco, Jossey-Bass Publishers, 1993. pp.14853.

Arends, R.; Balbás Diez-Barroso, C.; Vadillo, G. \& Klinger, C. Aprender a Enseñar. Ciudad de México, McGrawHill, 2007.

Bloom, B. S. Taxonomía de los Objetivos de la Evaluación. Buenos Aires, El Ateneo, 1971.

Brookhart, S. M. Developing measurement theory for classroom assessment purposes and uses. Educ. Meas. Issues Pract., 22(4):5-12, 2003.

Bricall, J. M. \& Brunner, J. J. Universidad Siglo XXI. Europa y América Latina. Regulación y Financiamiento. Paris, Documentos Columbus sobre Gestión Universitaria, Cinda-Columbus, 2000.

Crooks, T. J. The impact of classroom evaluation practices on students. Rev. Educ. Res., 58(4):438-81, 1988.

Himmel, E. Evaluación de aprendizajes en la educación superior. Rev. Investig. Educ. Latinoam., 33(2):199-211, 2011.

Laorden, C; García Barriocanal, E. \& Sánchez, S. Integrando descripciones de habilidades cognitivas en los metadatos de los objetos de aprendizaje estandarizados. Rev. Educ. Distancia, 4:14, 2005.

Palomba, C. A. \& Banta, T. W. Assessment Essentials: Planning, Implementing, and Improving Assessment in Higher Education. San Francisco, Josses-Bass Publishers, 1999.
Ríos, P. Claves para una Pedagogía Alternativa. Ponencia. Barquisimeto, Congreso Internacional de Pedagogía Alternativa, 2000.

Stiggins, R. New Assessment Beliefs for a New School Mission. Phi Delta Kappan, 86(1):22-7, 2004.

\author{
Dirección para Correspondencia: \\ Dr. Joel Bravo Bown \\ Departamento de Odontología \\ Facultad de Medicina y Odontología \\ Universidad de Antofagasta \\ Antofagasta \\ CHILE \\ Email: joel.bravo@uantof.cl \\ jbravo@odontologia.uchile.cl
}

Recibido: 31-03-2016

Aceptado: 22-08-2016 